\title{
Challenges and Progress towards Low Voltage Imaging in VPSEM
}

\author{
Brendan J Griffin
}

*Centre for Microscopy and Microanalysis, The University of Western Australia, 35 Stirling

Highway, Crawley, WA Australia 6009

An ongoing challenge in VPSEM is to collect high resolution and high signal:noise images using low voltage primary electron beams. Current generation VPSEM have excellent low voltage performance in high vacuum operating conditions with specifications around $1 \mathrm{~nm}$ at $15 \mathrm{kV}$ and $4 \mathrm{~nm}$ at $0.1 \mathrm{kV}$ yet offer only $2 \mathrm{~nm}$ at $30 \mathrm{kV}$ in VP mode. Comparative resolution data from secondary, backscattered and VP secondary detectors is given in table 1 illustrating this poorer performance . Better VP mode resolution through an innovative detector design has been reported at $5 \mathrm{kV}$ with imaging down to $2 \mathrm{kV}[1]$. Low voltage imaging in VPSEM with accelerating voltages down to $0.5 \mathrm{kV}$ has also been reported over the last decade but they have poor signal:noise and relatively low resolution (fig. 1) [2]. These data were collected at gas chamber pressures ranging up to 1.9 torr.

The problem is the scattering of the primary electron beam by the chamber gas, a well-documented function of the gas itself, and the operating parameters of primary electron energy, chamber gas pressure, and gas path length [3,4]. Most VP imaging is based on the use air or water vapour, for reasons of ionization efficiency, ability to maintain the state of hydrated samples, availability and/or cost, leaving chamber pressure and gas path length as the operating variables. To achieve the high resolution requirement a short working distance is essential and this also minimizes the gas path length. The latter effect is critical as simple modeling shows that the high primary scattering at low Eo will preclude imaging at anything but short gas path lengths (fig. 2).

The conflict that arises is that with a short working distance and gas path length the amplification of the emitted signal will be low and lead to poor signal;noise [2]. To overcome this aspect a side positioned, rather than top (pole-piece) positioned gaseous secondary electron detector (GSED) geometry allows an appropriate detector - sample spacing for maximum gas amplification of the signal and so offers the potential for improved low voltage imaging.

Initial imaging with an off-axis optically-based GSED have been successful down to $1 \mathrm{kV}$ at 0.2 torr using air as the imaging gas with a good signal:noise (fig.3). The resolution has been measured to $8 \mathrm{~nm}$ at $5 \mathrm{kV}$ on the first generation detector. Data will be presented on a recently completed second generation detector from a range of samples.

\section{References}

[1] W.R. Knowles et al., Microsc. Microanal. 10 (Suppl 2) (2004) 1060.

[2] B.J. Griffin et al., Scanning 17 (1995) 58.

[3] G.D. Danilatos J. Microscopy, 160 (1990) 9.

[4] B.L.Thiel ., Microsc. Microanal. 10 (Suppl 2) (2004) 128.

[5] This work was supported by funds from the NANO Major National Research Facility. Dr. D. Joy generously provided the Monte Carlo modeling software used. 


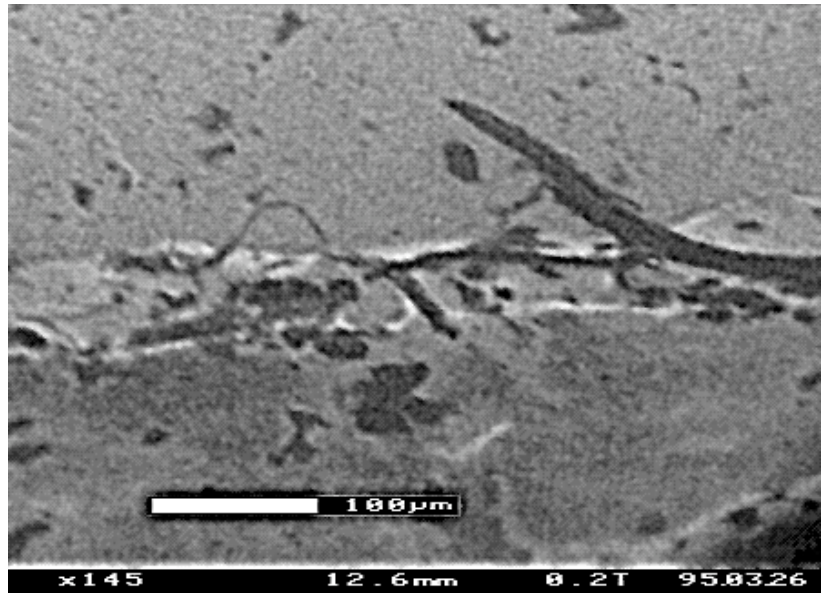

(a)

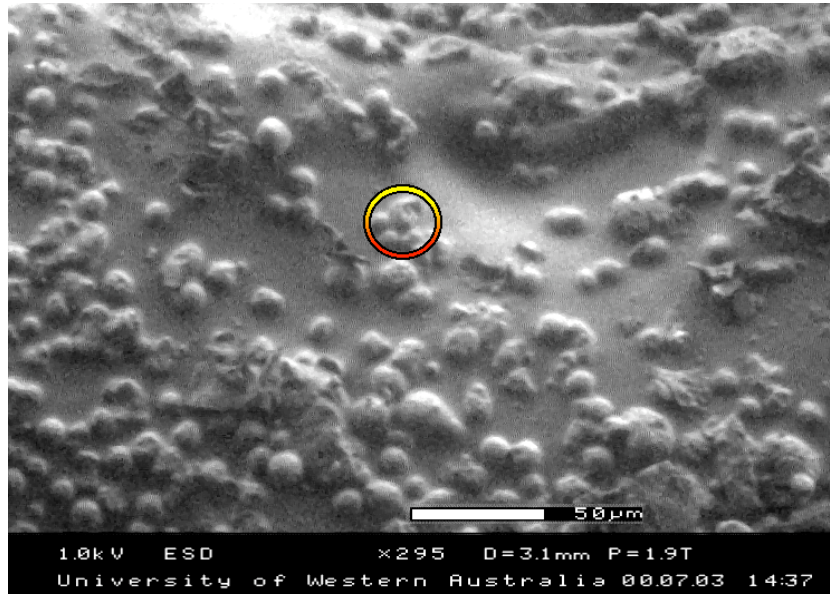

(b)

Figure 1: VPSEM images at low $\mathrm{E}_{\mathrm{o}}$ using a vertically positioned grid GSED of (a) carbon tape on aluminium collected at $0.5 \mathrm{kV}$ and 0.2 torr, and (b) tin spheres on carbon tape collected at $1.0 \mathrm{kV}$ and 1.9 torr. Both images obtained using an ElectroScan E-3 ESEM with water vapour.

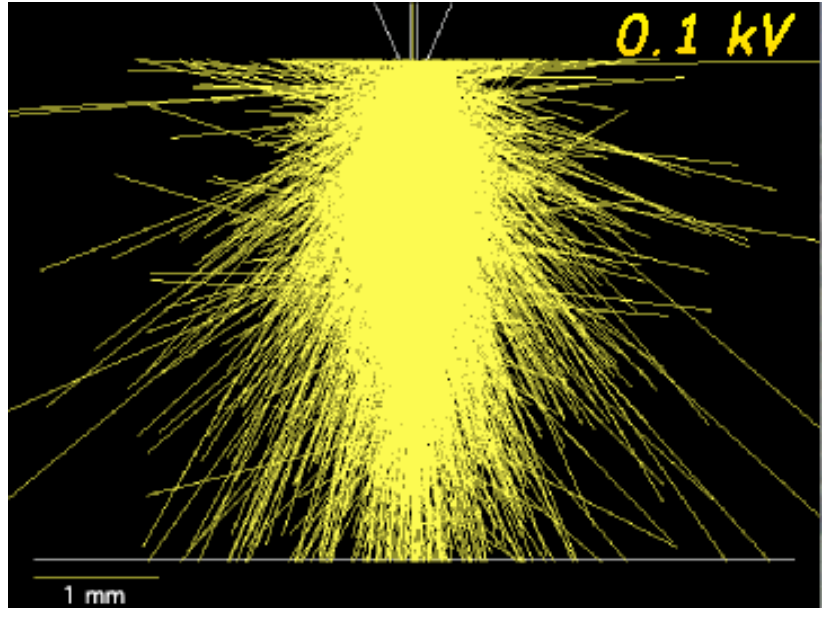

Figure 2: Primary beam scattering modeled for $\mathrm{E}_{\mathrm{o}}=0.1 \mathrm{kV}$, a gas path length of $4 \mathrm{~mm}$, and $\mathrm{a}_{2} \mathrm{O}$ gas chamber pressure of 2 torr

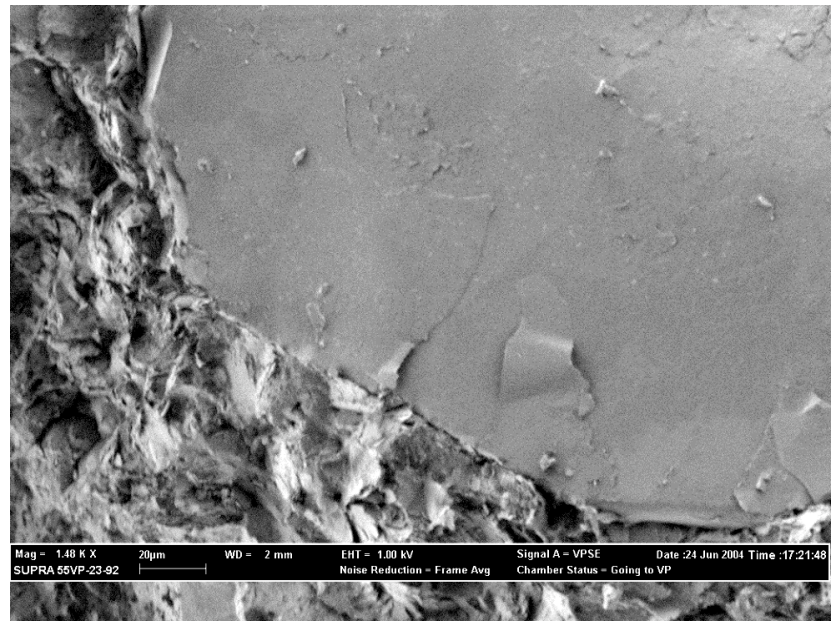

Figure 3: VPSEM image of a mica flake in a sandstone collected at Eo $=1 \mathrm{kV}, 2 \mathrm{~mm}$ GPL and 0.2 torr (air) using a side positioned opticallybased GSED in a Zeiss 1555VP SEM.

$\begin{array}{cccc}\text { Eo } & \text { se } & \text { bse } & \text { vpse } \\ 20 & 3.6 & 6.0 & 9.7 \\ 18 & 4.2 & 7.2 & 10.1 \\ 16 & 4.7 & 8.4 & 18.9 \\ 14 & 4.2 & 10.3 & 9.5 \\ 12 & 3.0 & 13.7 & 14.6 \\ 10 & 3.8 & 19.7 & 13.3 \\ 5 & & & 8.4\end{array}$

Table 1: Resolution measurement $(\mathrm{nm})$ variation against accelerating voltage (Eo) for different detectors under constant conditions. 